\title{
High-resolution confirmation of an extended helium atmosphere around WASP-107b
}

\author{
R. Allart ${ }^{1}$, V. Bourrier ${ }^{1}$, C. Lovis ${ }^{1}$, D. Ehrenreich ${ }^{1}$, J. Aceituno ${ }^{2,3}$, A. Guijarro ${ }^{2}$, F. Pepe ${ }^{1}$, D. K. Sing ${ }^{4,5}$, \\ J. J. Spake ${ }^{4,5,6}$, and A. Wyttenbach ${ }^{7}$ \\ ${ }^{1}$ Observatoire astronomique de l'Université de Genève, Université de Genève, 51 chemin des Maillettes, 1290 Versoix, Switzerland \\ e-mail: romain.allart@unige.ch \\ 2 Centro Astronomico Hispano Aleman, Sierra de los filabres sn, Gérgal, Almería, Spain \\ ${ }^{3}$ Instituto de astrofísica de Andalucía (CSIC), Glorieta de la Astronomia sn, Granada Spain \\ ${ }^{4}$ Department of Earth \& Planetary Sciences, Johns Hopkins University, Baltimore, MD, USA \\ ${ }^{5}$ Department of Physics \& Astronomy, Johns Hopkins University, Baltimore, MD, USA \\ ${ }^{6}$ Astrophysics Group, School of Physics, University of Exeter, Stocker Road, Exeter, EX4 4QL, UK \\ ${ }^{7}$ Leiden Observatory, Leiden University, Postbus 9513, 2300 RA Leiden, The Netherlands
}

Received 18 December 2018 / Accepted 22 January 2019

\begin{abstract}
Context. Probing the evaporation of exoplanet atmospheres is key to understanding the formation and evolution of exoplanetary systems. The main tracer of evaporation in the UV is the Lyman- $\alpha$ transition, which can reveal extended exospheres of neutral hydrogen. Recently, the near-infrared (NIR) metastable helium triplet (10833 $\mathrm{A}$ ) revealed extended thermospheres in several exoplanets. This opens a new window into evaporation.

Aims. We aim at spectrally resolving the first helium absorption signature detected in the warm Saturn WASP-107b with the Wide Filed Camera 3 on board the Hubble Space Telescope (HST/WFC3).

Methods. We obtained one transit of WASP-107b with CARMENES installed on the $3.5 \mathrm{~m}$ telescope at the Calar Alto observatory.

Results. We detect an excess helium absorption signature of $5.54 \pm 0.27 \%(20 \sigma)$ in the planet rest frame during the transit. The detection is in agreement with the previous detection achieved with HST/WFC3. The signature shows an excess absorption in the blue part of the lines, suggesting that He I atoms are escaping from the atmosphere of WASP-107b. We interpret the time-series absorption spectra using the 3D EVE code. Our observations can be explained by combining an extended thermosphere that fills half of the Roche lobe and a large exospheric tail sustained by an escape rate of metastable helium of about $10^{6} \mathrm{~g} \mathrm{~s}^{-1}$. In this scenario, however, the upper atmosphere needs to be subjected to a reduced photoionisation and radiation pressure from the star for the model to match the observations.

Conclusions. We confirm the presence of helium in the atmosphere of WASP-107b at high confidence. The helium feature is detected from space and from the ground. The ground-based high-resolution signal brings detailed information about the spatial and dynamical structure of the upper atmosphere, and simulations suggest that the He I signature of WASP-107b probes both its thermosphere and exosphere, establishing this signature as a robust probe of exoplanetary upper atmospheres. Surveys with NIR high-resolution spectrographs (e.g. CARMENES, the Spectromètre infrarouge (SPIRou), or the Near-Infrared Planet Searcher (NIRPS)) will deliver a statistical understanding of exoplanet thermospheres and exospheres through the helium triplet.
\end{abstract}

Key words. planets and satellites: atmospheres - planets and satellites: individual: WASP-107b - methods: observational techniques: spectroscopic

\section{Introduction}

Over the past decade, the exoplanetology field entered a phase in which exoplanet atmospheres are characterised in great detail. The easiest targets for atmospheric studies are hot planets that orbit close to their host stars, such as hot Jupiters and warm Neptunes, whose hydrogen- and helium-dominated atmospheres can reach large scale heights. Atmospheres heated through absorption of the stellar irradiation can expand hydrodynamically (Vidal-Madjar et al. 2003; Lammer et al. 2003). Hydrogen, through the Lyman- $\alpha$ line at UV wavelengths, was first observed to escape from hot Jupiters such as HD 209458b (Vidal-Madjar et al. 2003) and HD 189733b (Lecavelier Des Etangs et al. 2010) and to form extended cometary tails. More recently, even larger hydrogen exospheres were detected around the warm Neptunes GJ 436b (Ehrenreich et al. 2015) and GJ 3470b (Bourrier et al. 2018).
Warm Neptunes are important targets for atmospheric studies because many of them are located at the edge of the evaporation desert (Lecavelier Des Etangs 2007; Beaugé \& Nesvorný 2013). This desert is a lack of Neptune-mass planets at short orbital distance. It can be explained by planets that are not massive enough to retain their escaping gaseous atmosphere. Probing the gas that escapes from planets around the desert is thus particularly important to understand the evolution of the close-in planet population. While the Lyman- $\alpha$ line is an excellent tracer of the outermost atmospheric layers, it suffers from the lack of stellar continuum in the UV and from interstellar medium (ISM) absorption. This limits observations to extrasolar systems close to the Sun.

The metastable helium triplet, located in a spectral region that is devoid of strong ISM absorption (Indriolo et al. 2009, $10832.1,10833.2$ and $10833.3 \AA$ in the vacuum) with a bright continuum, was suggested to be a potential tracer of upper 
atmospheres (Seager \& Sasselov 2000; Oklopčić \& Hirata 2018) and has recently been detected in several exoplanets: the warm Saturn WASP-107b (Spake et al. 2018), the hot Jupiters WASP69b and HD 189733b (Nortmann et al. 2018; Salz et al. 2018), and the smaller warm Neptune HAT-P-11b (Allart et al. 2018; Mansfield et al. 2018). All these planets, except for WASP$107 \mathrm{~b}$, have been observed at high resolution with the Calar Alto high-Resolution search for M dwarfs with Exoearths with Near-infrared and optical Échelle Spectrographs (CARMENES), allowing us to trace the presence of He I down to the thermosphere.

WASP-107b (Anderson et al. 2017) is a warm Saturn $\left(0.11 \pm 0.01 M_{\mathrm{J}}\right.$ and $\left.0.94 \pm 0.02 R_{\mathrm{J}}\right)$ that is located at the upper radius border of the evaporation desert. It orbits a K6-type star with a period of 5.72 days (physical and orbital parameters are given in Table A.1). It has twice the mass of Neptune, but has a radius similar to Jupiter, and is thus one of the less dense exoplanets $\left(0.19 \pm 0.03 \mathrm{~g} \mathrm{~cm}^{-3}\right)$, which means that it is well suited for atmospheric characterisation. So far, two species have been detected in its atmosphere, water (Kreidberg et al. 2018) and helium (Spake et al. 2018), both with the Wide Field Camera 3 on board the Hubble Space Telescope (HST/WFC3). The helium feature, however, was unresolved and strongly diluted because it was measured with the G102 grism, which has a low resolution of $67 \AA$ (the typical width of the absorption helium feature is about $1 \AA$ ), and conservative $98 \AA$ bins have been used. Thus, the absorption profile of the He I absorption signature from WASP-107b remains poorly characterised. Observations of the helium triplet in WASP-107b at high spectral resolution are thus required to resolve the lines and constrain the properties of the metastable helium population around the planet. Here, we analyse one transit of WASP-107b obtained with CARMENES to spectrally resolve the helium triplet.

\section{CARMENES observations}

\subsection{Reduced data}

We observed one transit of WASP-107b on 23 February 2018 (DDT.S18.188; PI: Allart) with CARMENES installed on the $3.5 \mathrm{~m}$ telescope at Calar Alto (Quirrenbach et al. 2014). The transit dataset consists of 20 spectra with exposure times of $956 \mathrm{~s}$, with 10 spectra covering the $2.75 \mathrm{~h}$ of the transit. The signal-tonoise ratio $(\mathrm{S} / \mathrm{N})$ varies from 26 to 52 within the Echelle order 55 (10 804-11 005 $\AA$ ). Two spectra obtained at the end of the night were excluded from the following analysis because their $\mathrm{S} / \mathrm{N}$ is lower than 30 . Data were automatically reduced with the CARMENES Reduction and Calibration pipeline (Caballero et al. 2016), which applies a bias, flat, and cosmic ray correction of the raw spectra, and then a flat-relative optimal extraction (FOX; Zechmeister et al. 2014) and wavelength calibration (Bauer et al. 2015). The resulting output is defined in the Earth laboratory frame and is composed of wavelength (defined in vacuum), flux, and flux uncertainty maps (order vs. pixel number). Our analysis focuses on the order 55, which includes the helium triplet.

\subsection{Telluric correction}

Ground-based spectra are contaminated by the Earth's atmosphere, which manifests itself as telluric absorption lines (including water lines) and as telluric emission lines (such as $\mathrm{OH}$ ). Both sets of lines are present near the helium triplet in our observations (Fig. 1).
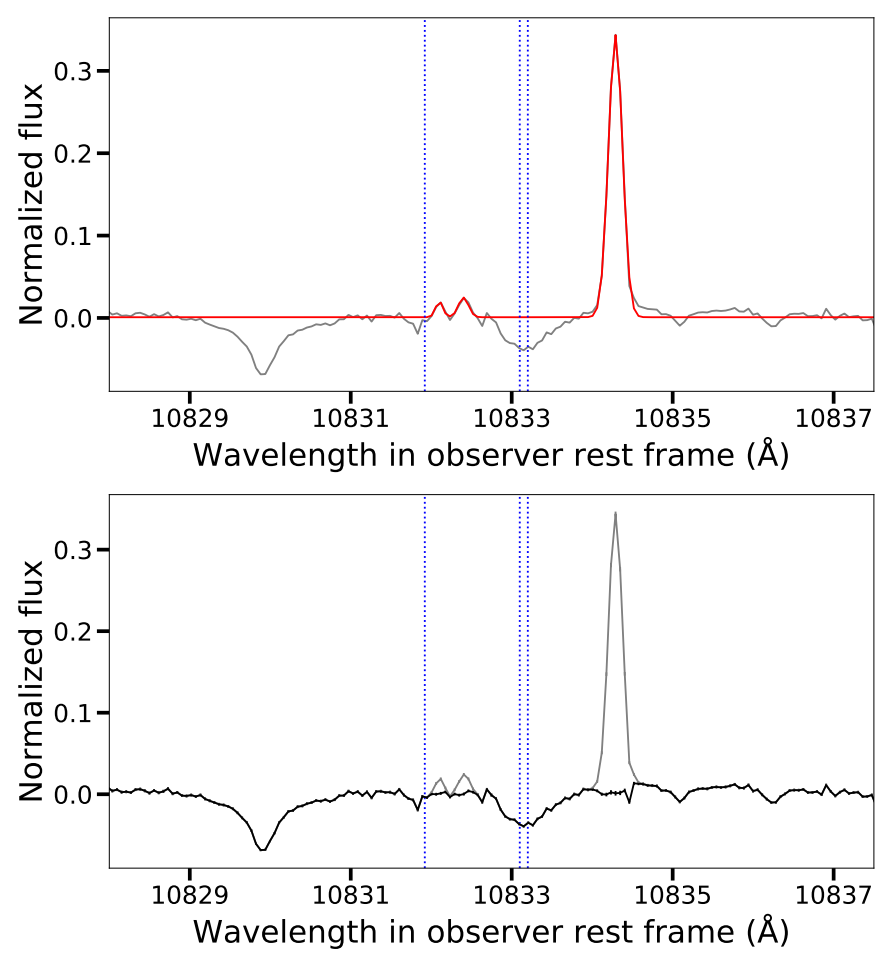

Fig. 1. Master-out stellar spectrum in the observer frame (grey profile). Blue dotted lines indicate the He I triplet. Top panel: best-fit to the telluric $\mathrm{OH}$ emission lines (red). Bottom panel: master-out spectrum corrected for the best-fit model of the $\mathrm{OH}$ lines (black profile).

The closest water absorption line is at $10835.1 \AA$ in the observer rest frame and is redshifted by more than $2 \AA$ from the planetary helium triplet (see Fig. 1). Because this is beyond the spectral range required for our analysis, we did not correct for this telluric line.

$\mathrm{OH}$ emission lines are clearly visible in Fig. 1 at 10832.1 , 10832.4 , and $10834.3 \AA$ and fall close to the stellar metastable helium lines at $10831.9,10833.1$, and $10833.2 \AA$ (wavelengths are given in the observer rest frame). During the observation the second fiber of the spectrograph, fiber B, was placed on the sky to monitor the emission lines. We built a high-S/N master-sky spectrum by co-adding all fiber B spectra. We confirmed the telluric origin of the $\mathrm{OH}$ lines, their wavelength positions, and we excluded the presence of other emission lines.

To correct for these emission lines, we built a master-out spectrum from out-of-transit spectra (phase $<-0.01263$ ) in the observer rest frame. We then fit a Voigt profile for the line at $10834.3 \AA$ and Gaussian profiles for the two shallowest lines (10 832.1 and $10832.4 \AA$ ). Finally, we fit the derived profiles to the emission lines in each individual exposure using a scaling factor. Best-fit models were then subtracted from each spectrum.

\section{Analysis of the observations}

We used a similar method as Allart et al. (2018) to build the transmission spectrum, which consists of Doppler-shifting the telluric-corrected spectra from the observer to the stellar rest frame by accounting for the barycentric Earth radial velocity, the stellar reflex motion of the star, and its systemic velocity (Table A.1). Then, spectra obtained out-of-transit are co-added to form a master-out spectrum, which is normalised to unity by reference bands in the blue (10821.9-10 827.1 $\AA$ ) and the red 


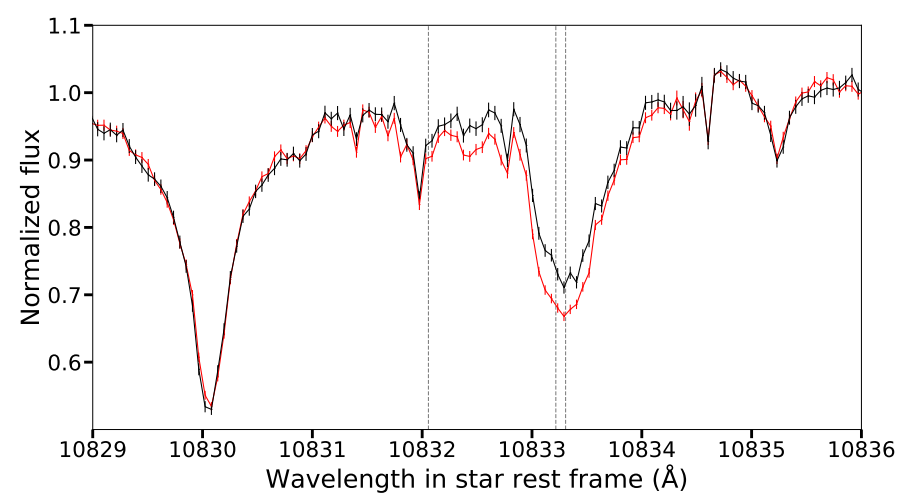

Fig. 2. Master-out (black) and master-in (red) spectra in the star frame. Vertical grey lines indicate the position of the helium triplet lines. In-transit absorption is clearly visible by eye.

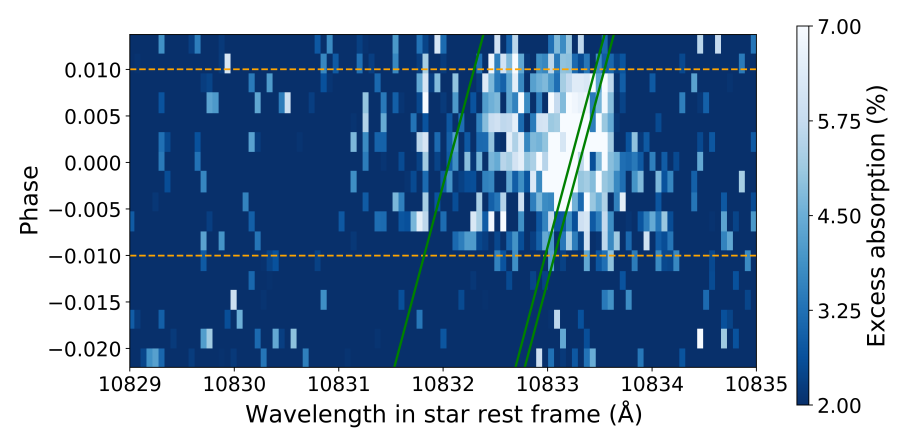

Fig. 3. Phase vs. wavelength map of ITS in the stellar frame. An excess absorption signature (in white) is visible during the transit (first and fourth contacts are highlighted in orange). Green curves show the helium planetary tracks.

(10 838.4-10 841.6 $\AA$ ) side of the helium triplet. Similarly, all spectra were normalised by the reference bands for comparison. Two spectra $(02 \mathrm{~h} 43$ and $03 \mathrm{~h} 02$ UT at phase -0.0032 and -0.0009 , respectively) exhibit emission features over 3 pixels from 10829.8 to $10830.3 \AA$, and one spectrum (03h53 UT, at phase 0.0053 ) also has an emission feature over 4 pixels from 10835.6 to $10836.0 \AA$. These features are likely due to cosmic hits. We replaced the flux value of these contaminated pixels by the median of the corresponding pixel over the night.

We first performed a visual comparison between the Masterout spectrum and a Master-in spectrum obtained by co-adding all normalised spectra between $t_{\text {II }}$ and $t_{\text {III }}$ (Fig. 2). An absorption signature is clearly visible by eye in the stellar spectrum, at the location of the helium triplet.

To further investigate the origin of the signature, we divided all spectra by the master-out and hereafter referred to them as individual transmission spectra (ITS). Figure 3 is a phase vs. wavelength map of the ITS in the stellar rest frame. The excess absorption signature is only visible during the transit and follows the radial velocity motion of the planet. This unambiguously shows the planetary origin of the helium absorption signature and confirms the unresolved detection from Spake et al. (2018).

The ITS were then scaled by the reference light curve and shifted to the planet frame. We used the Batman package (Kreidberg 2015) to build a reference light curve based on the transit depth, non-linear limb-darkening coefficients derived by Spake et al. (2018) from HST/WFC3 observations in the continuum around the helium triplet, and orbital properties derived by Anderson et al. (2017) and Dai \& Winn (2017; Table A.1).
To characterise the spectro-temporal properties of the helium absorption, we built a helium light curve, that is, the temporal variation of the flux in the spectral range absorbed by the planetary atmosphere, by integrating them from 10832.80 to $10833.55 \AA$. We also built a master transmission spectrum as the average of the seven ITS obtained between $\mathrm{t}_{\mathrm{II}}$ and $\mathrm{t}_{\mathrm{III}}$, selected to maximise the significance of the helium feature. The absorption depth from the atmospheric continuum was removed from this transmission spectrum (Fig. 4, top panel) to obtain the excess absorption spectrum by helium in the atmosphere.

Figure 4 shows the transmission spectrum and the helium light curve. There is a significant helium excess absorption of $5.54 \pm 0.27 \%$ (20- $\sigma$ detection) over a $0.75 \AA$ passband centred around the peak of excess absorption, which reaches $7.92 \pm 1.00 \%$ (or an equivalent opaque radius of $2.2 R_{\mathrm{p}}$ ) at $10833.1768 \AA$. The helium signature shows a strong spectral asymmetry, with excess absorption in the blue part of the lines. The helium light curve is roughly symmetrical in time and centred around mid-transit, but as the effective planet radius in the He I lines is larger than the atmospheric continuum, the transit duration is longer by about $30 \mathrm{~min}$. This further proves the He I lines of planetary origin because a false-positive signature arising from stellar activity does not change the transit duration.

The star WASP-107 is a slow rotator $\left(v_{\mathrm{eq}} \cdot \sin (i)=2.5 \mathrm{~km} \mathrm{~s}^{-1}\right.$, Anderson et al. 2017), and the Rossiter-McLaughlin (RM) of WASP-107b was not detected in velocimetry. The impact of the RM effect in the transmission spectrum is thus expected to be limited. We confirmed that it was below the $10^{-3}$ level by performing two simulations with the EVE code (see Sect. 4) assuming an obliquity of $\lambda=40^{\circ}$ or $\lambda=140^{\circ}$ (the range within which the obliquity of WASP-107b is currently known, Dai \& Winn 2017).

To compare our results with the HST low-resolution transmission spectrum of WASP-107b obtained by Spake et al. (2018), we convolved the CARMENES high-resolution transmission spectrum to the resolution of WFC 3 and binned it over $98 \AA$ pixels. Both transmission spectra were created using exposures obtained between phases -0.0063 to -0.0008 . The transmission spectra are compatible within the $1 \sigma$ errors (Fig. 5), and thus the signal is repeatable over two different epochs. WASP-107b is one of the few planets to have the same species detected in its upper atmosphere with both ground- and space-borne instruments.

\section{Interpretation of the $\mathrm{He}$ I signature}

Because it was not spectrally resolved, the signature of helium detected in the HST data of WASP-107b could be well fitted with two very different models for its upper atmosphere (Spake et al. 2018). A 1D model of a hot thermosphere extending far beyond the Roche lobe (based on Oklopčić \& Hirata 2018) yielded deep absorption lines that were symmetrical in the planet rest frame. In contrast, a 3D model of an exospheric tail (based on Bourrier \& Lecavelier des Etangs 2013) yielded shallow absorption lines that were spread over a wide spectral range because of the dynamics of helium atoms that are blown away from the planet. Our time series of high-resolution CARMENES spectra allows us to study the profile of the absorption signature and its evolution over the transit in detail (Fig. 3). As described above, the He I absorption lines are asymmetrical, with a clear excess absorption at blueshifted velocities in the planet rest frame (Fig. 4). This suggests that the outer atmospheric layers are being blown away from WASP-107b, forming a comet-like tail that seems at odds with the symmetry of the 

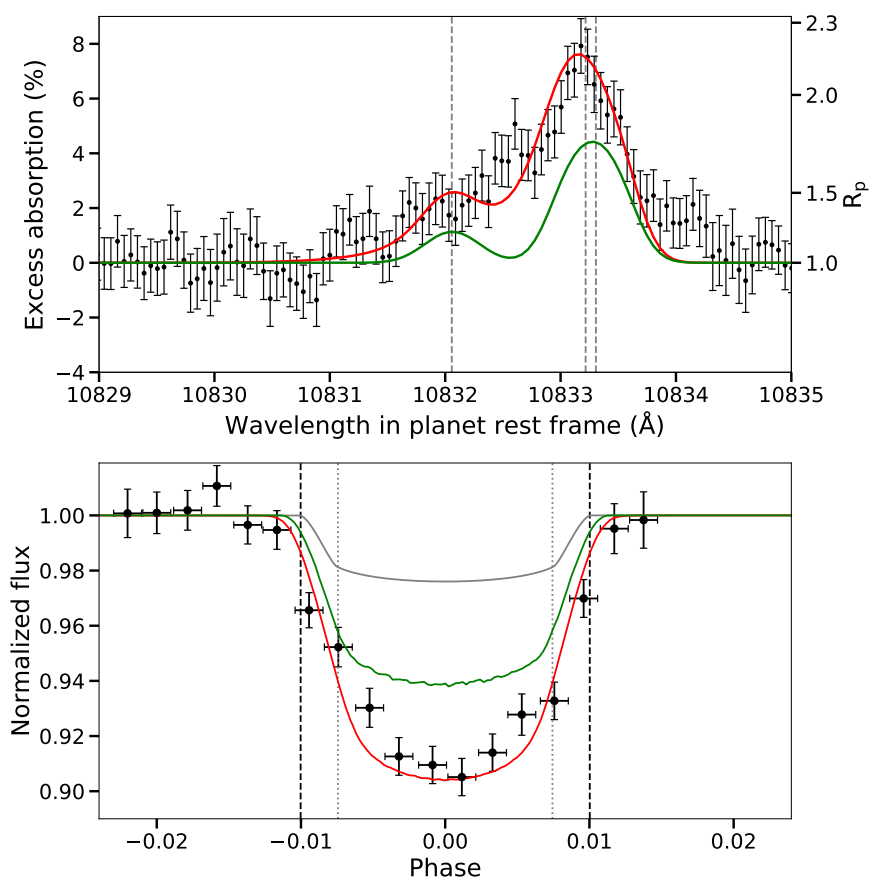

Fig. 4. Top panel: transmission spectrum of WASP-107b in the region of the He I triplet (black points) in the planet frame. The red line shows the theoretical profile obtained with the EVE code for the model shown in Fig 6. The green line shows the contribution from the model thermosphere alone. The three vertical grey dashed lines correspond to the helium triplet transition. Bottom panel: helium light curve integrated from 10832.80 to $10833.55 \AA$ from the observations (black), the theoretical atmospheric continuum (grey) obtained with Batman, and the simulated EVE atmosphere (same colour code as the top panel). The two vertical black dashed lines correspond to the contact points $t_{I}$ and $t_{I V}$, and the two vertical grey dotted lines correspond to $t_{\text {II }}$ and $t_{\text {III }}$.

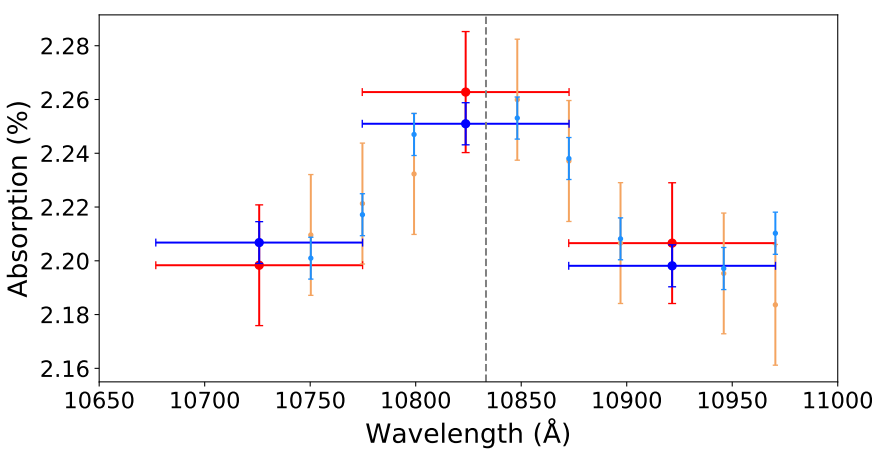

Fig. 5. Comparison between the HST/WFC3 dataset of Spake et al. (2018) in blue with our degraded high-resolution CARMENES dataset in red. Transmission spectra are binned over 98 A. Lighter points correspond to the same transmisssion spectra, but shifted by $24.5,49.0$, and $73.5 \AA$. The vertical grey dashed line is the helium triplet transition.

helium light curve (Fig. 4). This was expected from the 3D simulations performed in Spake et al. (2018), who showed that radiation pressure on escaping metastable helium atoms is so strong that a tail is formed that is aligned with the star-planet axis, with a roughly circular projection in the plane of sky. However, the measured absorption extends over a shorter wavelength range than predicted by the tail simulations from Spake et al. (2018), and it shows a deep core centred on the line transitions in the planet rest frame (Fig. 4). This similarity with the theoretical profile from the 1D model in Spake et al. (2018) suggests that part of the signal arises from helium in an extended thermosphere surrounding WASP-107b. Therefore, our observations might probe the thermosphere and exosphere of an exoplanet for the first time.

To further assess this possibility, we used the version of the EVaporating Exoplanet code (EVE; Bourrier \& Lecavelier des Etangs 2013; Bourrier et al. 2016) presented in Spake et al. (2018) and Allart et al. (2018). The planetary system is simulated in $3 \mathrm{D}$ in the stellar rest frame, and the code calculates theoretical spectra comparable to the CARMENES observations during the transit of the planet and its atmosphere. The thermosphere is modelled as a parametrised grid, using density and velocity profiles calculated with a spherically symmetric, steady-state isothermal wind model (Parker 1958; Oklopčić \& Hirata 2018). The exosphere is modelled by releasing metastable helium atoms at the top of the thermosphere, and computing their dynamics with Monte Carlo particle simulations that account for the planetary and stellar gravity and for the stellar radiation pressure. Metastable helium atoms in the simulation can be photoionised by the stellar incident radiation or be radiatively de-excited into their fundamental state. The density profile of metastable helium in the thermosphere is scaled so that it matches the density of exospheric metaparticles at the exobase.

Knowledge of the stellar flux is required below $2593 \AA$ to calculate the photoionisation rate of metastable helium atoms, and in the region of the $\mathrm{He}$ I triplet to calculate radiation pressure (Spake et al. 2018; Allart et al. 2018). In a first approach, we used the semi-synthetic spectrum of the K6-type star HD 85512 as a proxy for WASP-107, replacing it in the region of the He I triplet by the out-of-transit CARMENES spectrum rescaled to the flux level expected for a blackbody at the temperature of WASP-107. However, escaping atoms in the simulations were then accelerated so fast that they yielded excess absorption over a much wider velocity range than is observed in the data.

We found that artificially decreasing the stellar spectrum in the region of the $\mathrm{He}$ I triplet by a factor 50, so that radiation pressure is about $50 \%$ stronger than the star gravity, leads to the formation of an exospheric tail with a velocity gradient consistent with the data (Fig. 4). In this scenario, however, escaping helium atoms are subjected to a lower acceleration and are photoionised before they can reach the observed velocities. We were able to retrieve the observed shape of the absorption profile to a reasonable degree by decreasing the X-ray and extreme-ultraviolet (X-EUV, hereafter XUV) flux by the same factor as the NIR flux (Fig. 4). In that case, the lifetimes of metastable helium atoms become controlled by radiative deexcitation $(\sim 131 \mathrm{~min})$ rather than photoionisation $(\sim 7 \mathrm{~min}$ with the original XUV flux at the semi-major axis of $18.02 \pm 0.27 R_{*}$ ). It is possible that the XUV spectrum of HD 85512 is not a good proxy for WASP-107, especially since the EUV portion is derived empirically (France et al. 2016). However, we see no reason for the intrinsic stellar spectrum of WASP-107 to be lower than the expected blackbody in the region of the He I triplet. Our simulations thus suggest that additional physical processes shape the population of metastable helium atoms in the upper atmosphere of WASP-107b. For example, they might be shielded from incoming photons by an unkown absorber, or their dynamics could be affected by collisions with other escaping species or with stellar wind particles.

When we assume that simulations with a reduced photoionisation and radiation pressure capture the overall exosphere structure of WASP-107b, it is interesting to determine the upper atmospheric properties that are required to explain the data. The model in Fig. 6 was obtained by setting the 


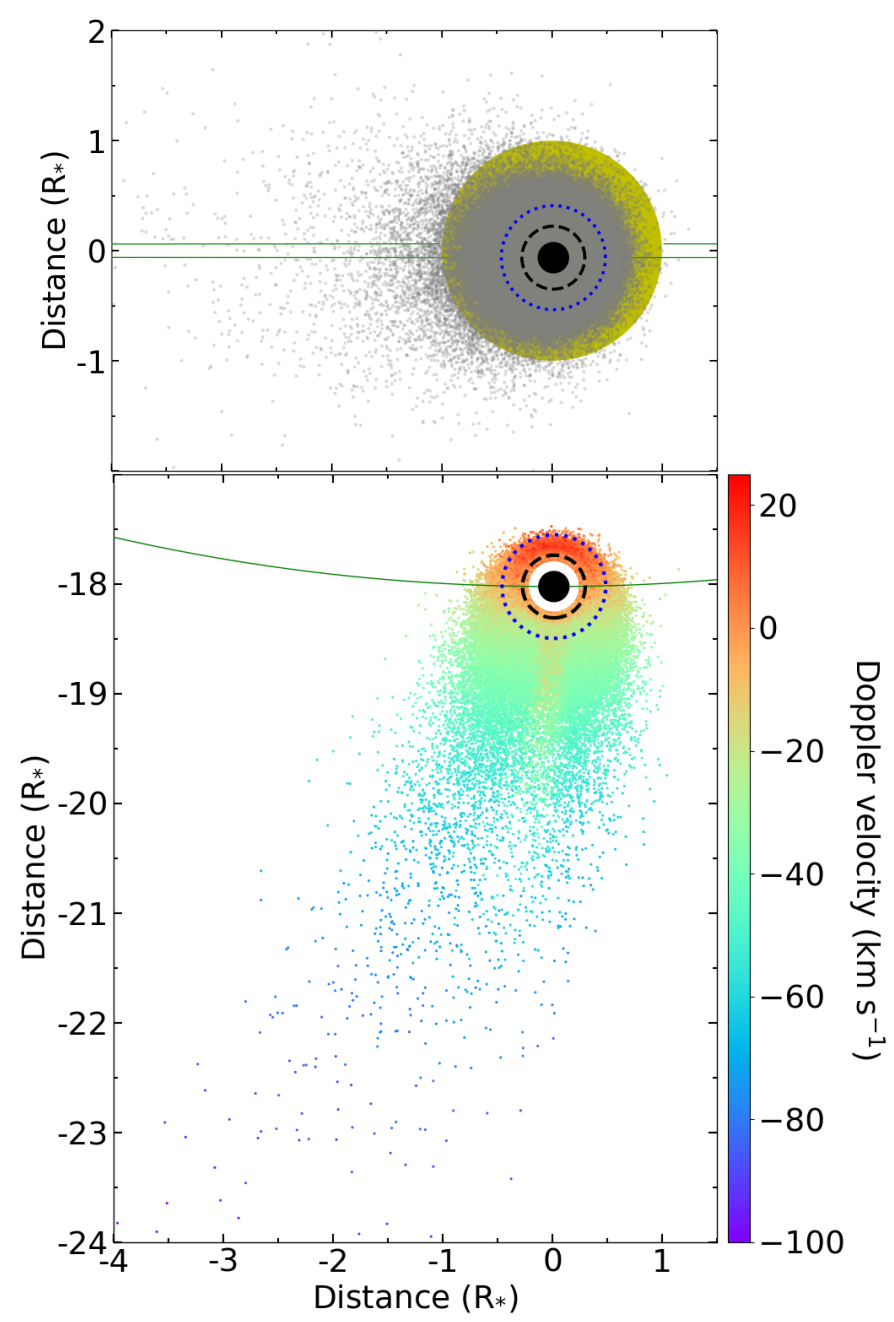

Fig. 6. 3D model of the upper atmosphere of WASP-107b simulated with the EVE code. Particles of metastable helium escape at the exobase (shown in projection as a dashed black circle), which separates the simulated thermosphere and exosphere. The planet as seen in the NIR continuum is shown as a black disc. The dotted blue line shows the projection of the Roche lobe. The green curve shows the orbit of WASP-107b. Top panel: view along the line of sight towards Earth, with metastable helium particles shown in grey. The yellow disc is the star. Bottom panel: view from above of the planetary system. Metastable helium particles are coloured as a function of their radial velocity and only shown within the orbital plane for the sake of clarity.

exobase at $2 R_{\mathrm{p}}$, so that thermosphere and exosphere both contribute to the theoretical absorption profile (Fig. 4). With a thermospheric temperature of $12000 \mathrm{~K}$ (assuming a solar-like hydrogen-helium composition with a mean atomic weight of 1.2), metastable helium atoms escape at the exobase with a thermal wind velocity of $\sim 12 \mathrm{~km} \mathrm{~s}^{-1}$. They fill the Roche lobe $\left(3.3 R_{\mathrm{p}}\right)$ before being blown away by radiation pressure (Fig. 6). The absorption profile was well reproduced with an escape rate of metastable helium of about $8 \times 10^{5} \mathrm{~g} \mathrm{~s}^{-1}$, which is in between the mass-loss rates derived from the HST data by the 1D thermospheric and 3D exospheric models in Spake et al. (2018).

\section{Conclusion}

Spake et al. (2018) have detected absorption in the He I triplet with HST during a single transit of the warm Neptune
WASP-107b. Because of the low spectral resolution of WFC3, the absorption line profile could not be resolved, and the exact origin of helium in the planet atmosphere remained unclear. The strong dilution of helium absorption signatures measured with HST/WFC3 limits its detection with this instrument to exoplanets with the deepest atmospheric transits, such as WASP-107b. Using ground-based CARMENES observations, we detected and resolved the signature of helium at high confidence $(5.54 \pm 0.27 \%, 20 \sigma)$ during a single transit of WASP-107b. The absorption signature is located at the He I triplet wavelengths in the planetary rest frame, displays a longer transit duration than the atmospheric continuum, and occurs during the planetary transit, which unambiguously shows its planetary origin and confirms the result of Spake et al. (2018).

WASP-107b is the second exoplanet for which helium has been detected from the ground and from space after HAT-P-11b (Allart et al. 2018; Mansfield et al. 2018). While the helium absorption shows no temporal asymmetry, its signature in the planet rest frame is asymmetric with excess absorption in the blue parts of the lines. Using 3D numerical simulations with the EVE code, we explained the observations as caused by extended thermosphere that sustains an exospheric comet-like tail. This scenario requires that escaping helium atoms are blown away by a reduced radiation pressure, suggesting that additional physical mechanisms are at play.

Further observations at high spectral resolution are required to obtain more insight into the dynamics and structure of the upper atmosphere of WASP-107b, but also to investigate the possible temporal variability of the signature (see the case of HAT-P-11, Allart et al. 2018). Our observations nonetheless clearly show that the NIR helium triplet can trace both the thermosphere and the exosphere, in contrast to observations of other exoplanets in this line (Allart et al. 2018; Nortmann et al. 2018; Salz et al. 2018). Combined with the new generations of NIR high-resolution spectrographs (e.g. CARMENES, the Spectromètre Infrarouge (SPIRou), and the Near-Infrared Planet Searcher (NIRPS)) and their large atmospheric surveys, the He I triplet will usher in a new era of statistical studies of extended exoplanet atmospheres.

Acknowledgements. We thank the anonymous referee for the careful reading and comments. We acknowledge the Geneva exoplanet atmosphere group for fruitful discussions. This work has been carried out within the frame of the National Centre for Competence in Research "PlanetS" supported by the Swiss National Science Foundation (SNSF). R.A., V.B., C.L., D.E., and F.P. acknowledge the financial support of the SNSF. This project has received funding from the European Research Council (ERC) under the European Union's Horizon 2020 research and innovation programme (project FOUR ACES; grant agreement No 724427). A.W. acknowledges the financial support of the SNSF by the grant number P2GEP2_178191. This work was based on observations collected at the Centro Astronòmico Hispano Aleman (CAHA), operated jointly by the MaxPlanck Institut fur Astronomie and the Instituto de Astrofisica de Andalucia (CSIC) under DDT proposal.

\section{References}

Allart, R., Bourrier, V., Lovis, C., et al. 2018, Science, 362, 1384

Anderson, D. R., Collier Cameron, A., Delrez, L., et al. 2017, A\&A, 604, A110

Bauer, F. F., Zechmeister, M., \& Reiners, A. 2015, A\&A, 581, A117

Beaugé, C., \& Nesvorný, D. 2013, ApJ, 763, 12

Bourrier, V., \& Lecavelier des Etangs A. 2013, A\&A, 557, A124

Bourrier, V., Lecavelier des Etangs, A., Ehrenreich, D., Tanaka, Y. A., \& Vidotto, A. A. 2016, A\&A, 591, A121

Bourrier, V., Lecavelier des Etangs, A., Ehrenreich, D., et al. 2018, A\&A, 620, A147

Caballero, J. A., Guàrdia, J., Lòpez del Fresno, M., et al. 2016, SPIE, 9910, $99100 \mathrm{E}$ 
Dai, F., \& Winn, J. N. 2017, AJ, 153, 205

Delisle, J.-B., Ségransan, D., Buchschacher, N., \& Alesina, F. 2016, A\&A, 590, A134

Díaz, R. F., Ségransan, D., Udry, S., et al. 2016, A\&A, 585, A134

Ehrenreich, D., Bourrier, V., Wheatley, P. J., et al. 2015, Nature, 522, 459

France, K., Loyd, R. O. P., Youngblood, A., et al. 2016, ApJ, 820, 89

Indriolo, N., Hobbs, L. M., Hinkle, K. H., \& McCall, B. J. 2009, ApJ,703, 2131

Kreidberg, L. 2015, PASP, 127, 1161

Kreidberg, L., Line, M. R., Thorngren, D., Morley, C. V., \& Stevenson, K. B. 2018, ApJ, 858, L6

Lammer, H., Selsis, F., Ribas, I., et al. 2003, ApJ, 598, L121

Lecavelier Des Etangs, A. 2007, A\&A, 461, 1185
Lecavelier Des Etangs, A., Ehrenreich, D., Vidal-Madjar, A., et al. 2010, A\&A, 514, A72

Mansfield, M., Bean, J. L., Oklopčić, A., et al. 2018, ApJ, 868, L34

Nortmann, L., Pallé, E., Salz, M., et al. 2018, Science, 362, 1388

Oklopčić, A., \& Hirata, C. M. 2018, ApJ, 855, L11

Parker, E. N. 1958, ApJ, 128, 664

Quirrenbach, A., Amado, P. J., Caballero, J. A., et al. 2014, SPIE, 9147, 91471F

Salz, M., Czesla, S., Schneider, P. C., et al. 2018, A\&A, 620, A97

Seager, S., \& Sasselov, D. D. 2000, ApJ, 537, 916

Spake, J. J., Sing, D. K., Evans, T. M., et al. 2018, Nature, 557, 68

Vidal-Madjar, A., Lecavelier des Etangs, A., Désert, J.-M., et al. 2003, Nature, 422, 143

Zechmeister, M., Anglada-Escudé, G., \& Reiners, A. 2014, A\&A, 561, A59

\section{Appendix A: Table}

Table A.1. Adopted physical and orbital parameters of WASP-107b.

\begin{tabular}{lccr}
\hline \hline Parameter & Symbol & Value & Reference \\
Stellar radius & $R_{*}$ & $0.66 \pm 0.02 R_{\odot}$ & Anderson et al. (2017) \\
Planet radius & $R_{\mathrm{p}}$ & $0.94 \pm 0.02 R_{\mathrm{J}}$ & Anderson et al. (2017) \\
White-light radius ratio & $R_{\mathrm{p}} / R_{*}$ & $0.142988 \pm 0.00012$ & Spake et al. (2018) \\
Stellar mass & $M_{*}$ & $0.69 \pm 0.05 M_{\odot}$ & Anderson et al. (2017) \\
Planet mass & $M_{\mathrm{p}}$ & $0.11 \pm 0.01 M_{\mathrm{J}}$ & This work, DACE \\
Epoch of transit & $T_{0}$ & $2457584.329897 \pm 0.000032 \mathrm{BJD}$ & Dai \& Winn (2017) \\
Duration of transit & $T_{14}$ & $0.1147 \pm 0.0003 \mathrm{~d}$ & Anderson et al. (2017) \\
Orbital period & $P$ & $5.721474 \pm 0.000004 \mathrm{~d}$ & Dai \& Winn (2017) \\
Systemic velocity & $\gamma$ & $14137.88 \pm 1.80 \mathrm{~m} \mathrm{~s}^{-1}$ & This work, DACE \\
Semi-amplitude & $\mathrm{K}_{*}$ & $16.45 \pm 1.21 \mathrm{~m} \mathrm{~s}^{-1}$ & This work, DACE \\
Eccentricity & $e$ & 0.0 & \\
Argument of periastron & $\omega$ & 0.0 & Fixed \\
Semi-major axis & $a$ & $18.02 \pm 0.27 R_{*}$ & This work, DACE \\
Inclination & $i$ & $89.8 \pm 0.2{ }^{\circ}$ & Dai \& Winn (2017) \\
Limb-darkening & $u_{1}$ & 0.72615373 & Spake et al. (2018) \\
Limb-darkening & $u_{2}$ & -0.70859436 & Spake et al. (2018) \\
Limb-darkening & $u_{3}$ & 1.09027178 & Spake et al. (2018) \\
Limb-darkening & $u_{4}$ & -0.45320191 & Spake et al. (2018) \\
\hline
\end{tabular}

We refined the planetary mass, the radial velocity semiamplitude, and the semi-major axis of the orbit using the DACE platform (Delisle et al. 2016; Díaz et al. 2016) based on the existing radial velocity data points obtained with Coralie and the High Accuracy Radial velocity Planet Searcher (HARPS). We used a Metropolis-Hastings Markov chain Monte Carlo algorithm with Gaussian priors based on previous studies (Anderson et al. 2017; Dai \& Winn 2017). 